\title{
MR Imaging of Papilledema and Visual Pathways: Effects of Increased Intracranial Pressure and Pathophysiologic Mechanisms
}

\author{
N. Passi, A.J. Degnan, and L.M. Levy
}

\begin{abstract}
SUMMARY: Papilledema, defined as swelling of the optic disc, frequently occurs in the setting of increased ICP and in a variety of medical conditions, including pseudotumor cerebri, sinus thrombosis, intracerebral hemorrhage, frontal lobe neoplasms, and Chiari malformation. Noninvasive imaging of the $\mathrm{ON}$ is possible by using MR imaging, with a variety of findings occurring in the setting of papilledema, including flattening of the posterior sclera, protrusion of the optic disc, widening of the ONS, and tortuosity of the ON. Early recognition of papilledema and elevated ICP is of paramount importance for ensuring restoration of vision. Newer advanced MR imaging techniques such as fMRI and DTI may prove useful in the future to assess the potential effects of papilledema on retinal and visual pathway integrity.
\end{abstract}

ABBREVIATIONS: ICP = intracranial pressure; IIH = idiopathic intracranial hypertension; ON = optic nerve; ONS =optic nerve sheath; $\mathrm{SAS}=$ subarachnoid space

$\mathbf{P}$ apilledema is the term used to describe optic disc swelling associated with ICP. ${ }^{1}$ While the pathogenesis of papilledema is not fully understood, recent studies have demonstrated a link between elevated ICP and the development of papilledema. The importance of papilledema as a useful indicator of increased ICP has long been recognized, with this phenomenon posited as early as the 1920s, with 1 pioneering ophthalmologist commenting, "While it is true that papilledema is a variable correlate of increased intracranial pressure, it is, nevertheless, a valuable guide to the necessity for surgical intervention." ${ }^{2}$ Since the first descriptions of papilledema on fundoscopic examination, a variety of diseases have been reported as presenting with papilledema. Modern evidence suggests that papilledema is caused by an increase in ICP that is transmitted to the SAS surrounding the ON, thereby interrupting the metabolic processes of the nerve and consequently leading to edema, ischemia, and eventual visual impairment or loss. The urgency of this condition and the need for its prompt recognition are as important now as they were in 1937 when Dr. Gordon Holmes addressed the Ophthalmological Society, saying: "As the essential etiological factor in papilloedema is increase in ICP, the relief of this pressure will lead to its disappearance and remove the risk of blindness or serious deterioration of vision if it can be effected before secondary changes have devel-

From the Department of Radiology, George Washington University Hospital, Washington, DC.

Please address correspondence to Lucien M. Levy, 901 23rd St NW, Washington, DC 20037; e-mail: llevy@mfa.gwu.edu

- Indicates open access to non-subscribers at www.ajnr.org

http://dx.doi.org/10.3174/ajnr.A3022 oped in the disc. It is therefore natural that those who are primarily concerned in the conservation of the patient's sight should demand early or immediate operation, for it is only by operation that permanent relief of pressure can be obtained." 3

Papilledema has gained increasing interest in recent years among neuro-ophthalmologists as the result of several clinical studies demonstrating that it may have not only diagnostic potential as a measure of increased $\mathrm{ICP}^{4-7}$ but also therapeutic potential as a measure of disease severity and response to treatment. ${ }^{8}$

In attempting to detect and diagnose papilledema as early as possible in patients, MR imaging is becoming a useful noninvasive method. Because MR imaging can provide gross visualization of the optic globe, ON, orbits, and optic tract, ${ }^{9}$ it is an ideal tool to study the details of papilledema. This review provides a brief outline of the common MR imaging findings of papilledema and its pathologic mechanisms.

\section{ON ANATOMY}

The $\mathrm{ON}$ is a white matter tract of the CNS that passes through the optic canal into the orbit. Anatomically, the nerve can be divided into orbital and intracanalicular components. The orbital portion consists of the bulbar segment and the midorbital segment. The bulbar segment forms the widest part of the orbital portion and is adjacent to the ocular globe. The midorbital segment follows the bulbar segment. ${ }^{10}$

\section{Perioptic Meninges}

The perioptic meninges or ONS envelope the length of the ON up to the globe and is continuous with the intracranial meninges. For this reason, pressure changes in the intracranial space can be 
transmitted to the optic papilla via the SAS accompanying the ONS. $^{11}$

\section{SAS of the ON}

The SAS of the ON is a multichambered tubular system with a blind end behind the globe. The SAS of the ON is anatomically classified into a bulbar segment, intraorbital segment, and canalicular portion. These divisions are not uniform in architecture: The bulbar segment consists of trabeculae, the intraorbital segment consists of septa and pillars, and the canalicular portion consists of trabeculae and pillars. ${ }^{10}$ This architectural meshwork may play an important role in maintaining the pressure within the nerve.

\section{MR IMAGING FINDINGS}

A number of studies have used imaging techniques to investigate the anatomic changes of the $\mathrm{ON}$ in papilledema. ${ }^{5,6,8,9,12-15}$ Of the many imaging techniques, MR imaging has been of particular interest because of its ability to provide gross visualization of the optic globe, ON, orbits, and optic tract. Additionally, MR imaging provides higher soft-tissue contrast and free section orientation capabilities compared with CT and appears to be more accurate in assessing the ON than sonography. ${ }^{16}$

Despite these advantages, the $\mathrm{ON}$ has been technically difficult to image because of its small size: It is $0.4-0.6 \mathrm{~cm}$ in diameter within the orbit. ${ }^{8}$ T2-weighted FSE sequences with fat-suppression have been found to be optimal for visualizing the ONs and perioptic CSF. ${ }^{6,17-19}$ Coronal image acquisition is optimal for visualizing the true dimensions of the $\mathrm{ON}$ and perioptic CSF relative to the surrounding sheath. ${ }^{12}$ The most commonly reported macroscopic findings in MR images of patients diagnosed with papilledema are the following: 1) enlargement of the ONS, 5,6,8,9,20 2) flattening of the posterior sclera, ${ }^{13-15} 3$ ) protrusion of the optic papilla into the globe, ${ }^{6,11}$ and 4) tortuosity of the $\mathrm{ON}^{6}$

\section{Enlargement of the ONS}

The SAS around the ON in the orbit can be observed by using T2-weighted MR imaging with fat-saturation pulse sequences (Fig 1). The ONS diameter can be evaluated by measuring the outer diameter of the SAS. ${ }^{5}$

The normal ONS diameters just behind and $4 \mathrm{~mm}$ posterior to the globe are $5.52 \pm 1.11$ and $5.2 \pm 0.9 \mathrm{~mm}$, respectively. ${ }^{5}$ The ONS is widest anteriorly behind the globe and narrowed toward the orbital apex ${ }^{8}$; these dimensions are consistent with the results of a histologic study of the ON. ${ }^{12}$ The sheath of the ON behind the globe is the most distensible part of the ONS, giving it a bulbous appearance. $^{12}$

ONS enlargement appears as a widened ring of CSF around an $\mathrm{ON}$ and as a widened CSF signal intensity on either side of the $\mathrm{ON}$ on axial images. ${ }^{6}$ The finding of ONS enlargement have been reported previously in association with intracranial hypertension on sonography ${ }^{4}$ and CT. ${ }^{21}$ Seitz et $\mathrm{al}^{9}$ used MR imaging to examine patients with papilledema and found that the mean width of the ONS directly behind the globe was $7.54 \mathrm{~mm}( \pm 1.05 \mathrm{~mm})$ in pathologic conditions, compared with $5.52 \mathrm{~mm}( \pm 1.11 \mathrm{~mm})$ in healthy subjects. In addition, the CSF surrounding the ON from the optic globe toward the optic chiasm was visible over a longer
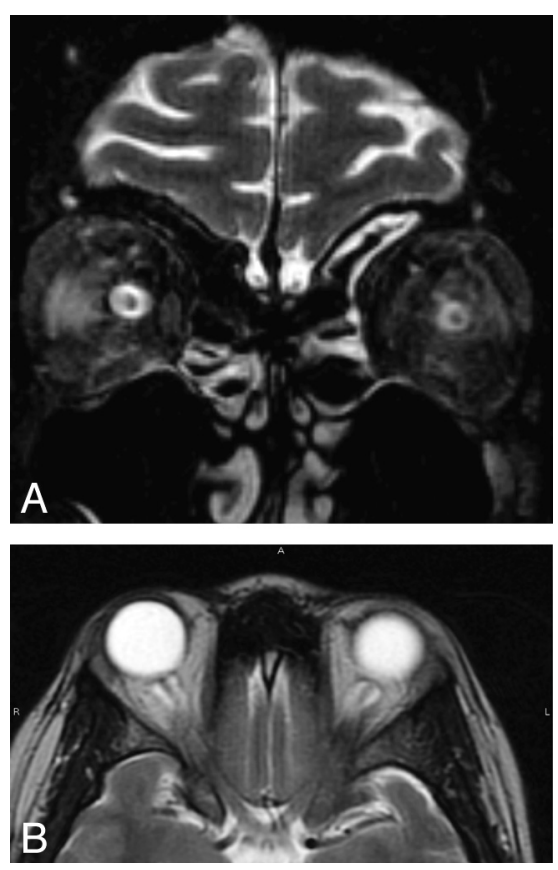

FIG 1. ONS enlargement. $A$, Coronal T2-weighted FSE image demonstrates bilateral widening of the ONS in a 32-year-old woman who presented with acute vision loss and headaches and was found to have papilledema on fundoscopic examination. The ONSs are abnormally increased in size (TR, $6816.7 \mathrm{~ms} / \mathrm{TE}, 84 \mathrm{~ms}$; 3-mm thickness; FOV, $18 \mathrm{~cm}$; matrix, $256 \times 224 ; 4$ excitations). B, Axial T2-weighted FSE image demonstrates the widening of the ONS bilaterally in a 31-year-old woman with known papilledema presenting with headaches (TR, 4400 ms/TE, 80 ms; 5-mm thickness; 6-mm spacing; FOV, 24 cm; $320 \times 256$ matrix).

distance $(12.4 \mathrm{~mm})$ in patients compared with healthy subjects $(6.3 \mathrm{~mm}){ }^{9}$ Similar findings were noted in patients with $\mathrm{IIH}$, with an enlarged and elongated SAS around the ON. ${ }^{8}$ The orbital portion of the ON was examined in patients at different stages of papilledema, and the diameter of the ONS was found to be increased compared with that in healthy subjects. ${ }^{20}$ With severe papilledema, the diameter of the ON just behind the globe was significantly less than that with moderate papilledema; this difference indicates that increased subarachnoid pressure may lead to gradual atrophy of the ON. ${ }^{20}$ Dilation of the SAS, compression of the $\mathrm{ON}$, and widening of the ONS were also observed with optic disc pallor. ${ }^{12}$ Broadening of the ON directly behind the optic globe may also occur with papilledema. ${ }^{9}$

MR imaging has been used to evaluate the effectiveness of treating elevated ICP with ONS fenestration by assessing the resolution of the optic sheath enlargement. ${ }^{5,8}$ ICP and ONS diameter in patients with chronic subdural hematoma or hygroma who underwent burr-hole craniotomy and continuous drainage have shown significant correlation. ${ }^{5}$ ONS diameter before surgery (6.1 $\mathrm{mm})$ was significantly reduced after surgery $(4.8 \mathrm{~mm}) .^{5}$ These findings suggest that the ONS diameter is a strong indicator of increased ICP. ${ }^{5,8}$

\section{Flattening of the Posterior Sclera}

Several studies have demonstrated posterior scleral flattening (Fig 2) in patients with elevated ICP. ${ }^{13-15}$ Gibby et al ${ }^{13}$ noted this finding on CT scans and considered it to be the mildest in the spec- 

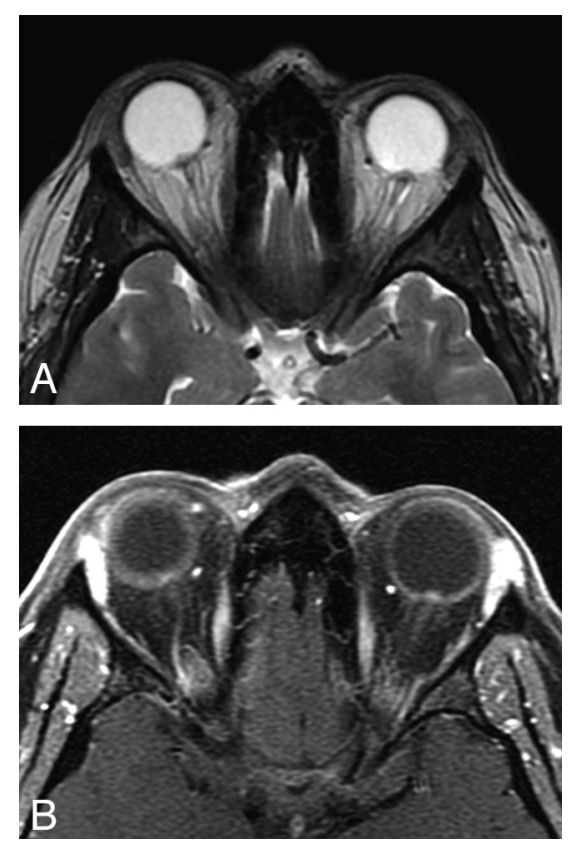

FIG 2. Optic papilla protrusion. In this T2-weighted axial image, both ON papillae protrude into the vitreous space of the globe. This 32year-old woman had experienced headaches and vision loss on presentation; she was also found to have papilledema on fundoscopic examination (same patient as in Fig 1A). A, Axial T2-weighted image demonstrates bilateral ON head protrusion (TR, $3000 \mathrm{~ms} / \mathrm{TE}, 84 \mathrm{~ms}$; 5-mm thickness; FOV, $24 \mathrm{~cm}$; matrix, $320 \times 256$; 1 excitation). $B$, Corresponding axial Tl-weighted postcontrast image demonstrates enhancement of the ON heads (TR, $766.7 \mathrm{~ms} / \mathrm{TE}, 9 \mathrm{~ms}$; 3-mm thickness; FOV, $18 \mathrm{~cm}$; matrix, $320 \times 191 ; 1$ excitation).

trum of changes leading to the protrusion of the ON head into the globe. Atta and Byrne ${ }^{14}$ noted flattening of the posterior sclera on sonography in some patients with choroidal folds and papilledema. Jacobson ${ }^{15}$ found bilateral flattening of the posterior sclera and distension of the perioptic SAS on MR imaging in a patient with elevated ICP and unilateral papilledema. These findings suggest that the combination of acquired hyperopia and choroidal folds may indicate pseudotumor cerebri in rare patients whose distal ONs are structurally resistant to developing papilledema.

\section{Protrusion of the Optic Papilla into the Globe}

The optic papilla is considered by some to be the site most vulnerable to the effects of elevated CSF pressure in the nerve sheath. ${ }^{17}$ Normally, the optic papilla appears as a flat hyperintense (hypointense relative to the vitreous of the globe of the eye) region in the posterior sclera. In pseudotumor cerebri, intraocular protrusion of the optic papilla is occasionally observed on MR imaging. Brodsky and Vaphiades ${ }^{6}$ found prelaminar enhancement in $50 \%$ of MR images of patients with pseudotumor cerebri. However, they also found an absence of prelaminar enhancement in some patients with florid papilledema. Intraocular protrusion was also demonstrated by Jinkins et $\mathrm{al}^{11}$ in 10 (67\%) of 15 patients with pseudotumor cerebri on MR imaging.

Intraocular protrusion of the optic disc (Fig 3) may not produce a sufficient signal-intensity differential with the vitreous of the globe on routine MR imaging. However, contrast-enhanced MR imaging may demonstrate a focal hyperintensity in the region

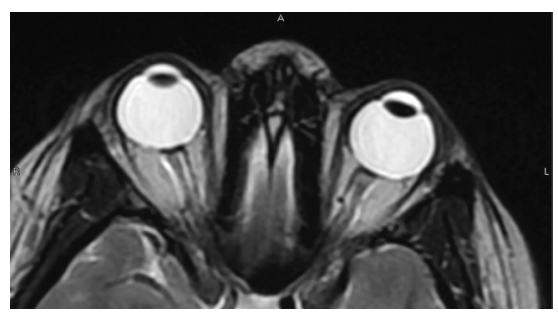

FIG 3. Posterior globe flattening. Flattening of the posterior globe is thought to occur in the same setting as papilledema with increased ICP. In this image, the site of the optic papilla is flattened and the normal globe contour is lost. This 37-year-old woman presented with headache and a sensation of head pressure. She was found to have papilledema and increased CSF opening pressure on lumbar puncture. (TR, $5650 \mathrm{~ms} / \mathrm{TE}, 88 \mathrm{~ms} ; 5-\mathrm{mm}$ thickness; FOV, $24 \mathrm{~cm} ; 320 \times 256$ matrix).

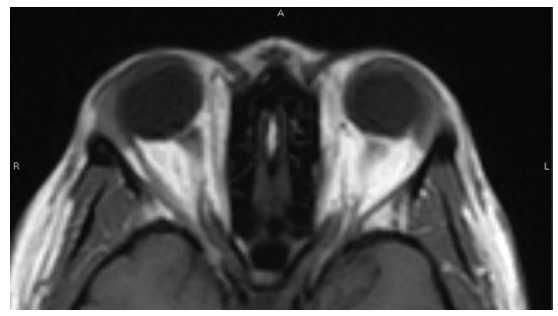

FIG 4. ON tortuosity. Bending of the ON can be seen more prominently in the right ON in this 40-year-old woman with history of headache diagnosed with increased ICP. In this particular case, there is a smear sign, in which orbital fat obscures part of the tortuous ON (TR, $620 \mathrm{~ms} / \mathrm{TE}, 9 \mathrm{~ms} / \mathrm{Tl}, 0 \mathrm{~ms}$; 5-mm thickness; 6-mm spacing; FOV, $24 \mathrm{~cm}$; $256 \times 192$ matrix).

of papilla protrusion because intraocular protrusion of the optic papilla disrupts blood flow in the optic prelaminar capillaries. ${ }^{6}$ The optic papilla usually appears hypointense relative to the vitreous fluid of the globe on T2-weighted images because it is largely composed of myelinated axons. However, extracellular edema generally produces increased signal intensity on T2weighted images, and this seems to contradict active capillary leakage. Histologic studies in patients with papilledema have demonstrated that despite capillary leakage, prelaminar axons appear distended but show little evidence of extracellular edema. This could explain the hypointensity of the optic papilla relative to the vitreous on T2-weighted images. ${ }^{11}$

\section{Tortuosity of the ON}

ON kinking or tortuosity (Fig 4) has also been associated with increased ICP. The ON tortuosity in patients with elevated ICP is attributable to the fixation of the distal and proximal points of the ON. The sensitivity of observing ON tortuosity in either the vertical or horizontal planes also depends on section thickness. The ability of axial MR imaging to display relatively minor degrees of horizontal tortuosity makes it a somewhat nonspecific finding. Tortuosity of the vertical component, which requires a greater deflection of the $\mathrm{ON}$ to be visible on axial scans, retains a higher specificity value. Vertical tortuosity of the ON is often accompanied by a "smear sign," in which the midportion of the ON appears obscured by a "smear" of orbital fat on T1-weighted images (Fig 4). ${ }^{6}$

Brodsky and Vaphiades ${ }^{6}$ theorized that perioptic distension would be expected to straighten the ONS if the globes were not 
tethered in the orbits by their rectus muscles and check ligaments. However, the absence of proptosis in patients with papilledema suggests that the focal pressure exerted by the bulbous portion of the distended perioptic nerve sheaths on the posterior sclera is insufficient to displace the globes anteriorly. Instead, the globe provides resistance to the distal bulbous portion of the ONS, which may kink the inflated ONS. ${ }^{6}$

\section{PATHOGENESIS OF PAPILLEDEMA}

Because all CSF spaces communicate freely, the pressure and composition of the CSF is thought to be the same throughout the CNS. Consequently, researchers have based their theories and experiments on the assumption that the pressure in the SAS surrounding the $\mathrm{ON}$ is the same as that in the cerebral and spinal SAS. However, this assumption has not been proved, mainly due to the difficulty in obtaining accurate pressure measurements in the SAS of the ON in vivo. In the SAS of the ON, CSF flows from the chiasmatic cistern through the canalicular portion and into the intraorbital portion of the $\mathrm{ON}$, a space that becomes a cul de sac at the back of the globe. ${ }^{1}$

A number of theories have been postulated during the past century to explain the pathogenesis of papilledema. In 1911, Leslie Paton, an ophthalmologist and Gordon Holmes, a neurologist, reported the results of the first definitive light microscopic study of papilledema. They concluded that papilledema was due to compression of the subarachnoid portion of the central retinal vein by a high ICP and that this produced dilation of the optic disc segment of the vein. Leakage from this dilated vein and its feeding capillaries then caused prelaminar optic disc swelling and bending of axons, which occasionally resulted in their fracture. ${ }^{22}$

In the late 20th century, the theory of axoplasmic stasis replaced the Paton-Holmes venous compression doctrine following electron microscopy evidence of intra-axonal edema of the optic disc in acute glaucoma, ocular hypotony, and increased ICP. Studies of axoplasmic flow within the ON, as measured by the progress of titrated leucine injected into the vitreous cavity and incorporated into retinal ganglion cells, showed that axoplasm was arrested in the region of the lamina cribrosa. It was thought that the high-pressure gradient, present in all 3 conditions, caused or contributed to the axoplasmic stasis. ${ }^{23-25}$

Currently, investigators are focusing on uncovering the mechanism by which elevated ICP causes papilledema. Two competing theories are at the forefront of current research — the mechanical theory and the ischemic theory. The mechanical theory postulates that elevated ICP causes direct compression of axons. The high ICP compresses the $\mathrm{ON}$ at its junction with the globe and distends its prelaminar axons as their axoplasm piles up at the scleral lamina. ${ }^{22}$ Howden et $\mathrm{al}^{26}$ found that CSF velocity is substantially slower in areas farther away from its inlets and that at certain sites, the flow nearly stagnates. Therefore, any reduction in CSF flow rate should first affect the SAS of the ON, which is farther away from the ventricles and anatomically does not allow continuous flow. $^{26}$

The ischemic theory postulates that elevated ICP causes reduced perfusion of the $\mathrm{ON}$ axons. The retrolaminar region of the $\mathrm{ON}$ is a watershed area and is prone to ischemic insult. ${ }^{22}$ With severe dilation of the distal optic SAS, all the regional neural and vascular structures can become compressed, compromising vascular flow. Trobe ${ }^{22}$ proposed that papilledema results primarily from the competitive relationship between the ciliary arterial circle and the choroidal circulation. If the subarachnoid pressure rises, the ciliary circulation becomes compressed and the arterial blood supply to the laminar $\mathrm{ON}$ is reduced, thereby interfering with the high flow to the choroid. The ON axons then become distended as chronic ischemia interferes with metabolic axoplasmic flow. ${ }^{22}$ In addition, if the pressure in the SAS of the ON is the same as that in the ventricular system, then the pial septal blood supply to the ON might become compromised by pressure, leading to hypo-oxygenation of the axons. With axoplasmic stasis, local toxic CSF metabolites may accumulate. ${ }^{1}$

\section{USING SIGNS OF PAPILLEDEMA TO DIAGNOSE ELEVATED ICP}

While researchers have investigated the relationship between elevated ICP and papilledema, they have also used ocular and ON abnormalities to diagnose elevated ICP. ${ }^{27}$ The ophthalmoscopic appearance of IIH is most often characterized by bilateral ON head swelling, but this can be subtle and variable. ${ }^{28-33}$ Furthermore, papilledema may be asymmetric or unilateral, ${ }^{33,34}$ and the degree of ON head swelling is poorly correlated with ICP.

In the recent William F. Hoyt Lecture of the American Academy of Ophthalmology, Dr Jonathan Trobe posited that papilledema is only a reliable indicator of chronically high ICP because the development of papilledema tends to lag behind the rise in ICP. Trobe noted that fewer than $20 \%$ of patients examined within a few days of head trauma or ruptured aneurysm have papilledema and only $6 \%$ of patients with chronically high ICP lack papilledema. ${ }^{22}$ On the other hand, intracranial hypertension can occur without the presence of papilledema. ${ }^{28-32,35}$ Possibly, MR imaging could assess the presence of intracranial hypertension before the development of papilledema. According to Hansen and Helmke, ${ }^{4}$ there is a correlation between the width of the ONS and increased ICP. A width of $>5 \mathrm{~mm}$ in this location is considered abnormal. ${ }^{9}$ This may occur because the ONS is not as rigid as other intracranial meningeal structures and can thus react without volume changes of intracranial CSF spaces. ${ }^{4}$

Elevated ICP may lead to several abnormalities on MR imaging, including the following: 1) flattening of the posterior sclera, 2) enhancement of the prelaminar $\mathrm{ON}, 3$ ) distension of the perioptic SAS, 4) intraocular protrusion of the prelaminar ON, 5) vertical tortuosity of the orbital ON, and 6) empty sella. ${ }^{6}$ In patients with pseudotumor cerebri, all neuroimaging signs except for intraocular protrusion of the optic disc have been found to be highly significant for the presence of elevated ICP. ${ }^{6}$ Flattening of the posterior sclera was found in $80 \%$ of these patients; empty sella, in $70 \%$; distension of the perioptic SAS, in $45 \%$; and enhancement of the prelaminar ON, in $30 \%$. On the basis of these signs, elevated ICP may be predicted in $90 \%$ of these cases. Posterior scleral flattening and prelaminar enhancement were thought to be confirmatory for papilledema, whereas distension of the perioptic nerves or empty sella was only considered suggestive for elevated ICP. ${ }^{6}$

Once the diagnosis of elevated ICP is established, the appearance of the discs and the severity of papilledema are commonly 
used as measures of disease severity and response to therapy. However, the degree of papilledema does not predict the severity of symptoms. Increased CSF pressure might produce different disc abnormalities depending on the normal size of the ONS. ${ }^{8}$ Further studies should lead to a better understanding of the mechanisms and augment our ability to detect papilledema on imaging and allow early intervention to maintain or restore vision.

\section{FUTURE DIRECTIONS OF MR IMAGING IN PAPILLEDEMA IMAGING}

MR imaging findings in papilledema only represent 1 aspect of papilledema-the anatomic alterations of the optic disc and nerve. Recent advances in MR imaging make it possible to more closely examine the interface between anatomic disruption and physiologic dysfunction. ${ }^{36}$ Theoretically, MR imaging could be used to predict the visual outcome of papilledema as a prognostic test-deficits in functional imaging of the retina or disruption of the optic pathways could suggest a greater risk of vision loss.

A number of advanced MR imaging techniques to enhance image quality have been developed in the past decade $e^{9,37,38}$ and have been found to be valuable in imaging the ON. ${ }^{39}$ High-resolution MR imaging may differentiate papilledema from other optic neuropathies and diagnose it earlier. High-resolution MR imaging has been used to visualize the $\mathrm{ON}$ and the smaller structures of the globe, including the lens, ciliary body, choroid, sclera, and retina with a high level of anatomic detail. ${ }^{40-44}$

fMRI has been used in mapping the function of cortical regions in a variety of healthy and disease states involving vision. ${ }^{45,46}$ In cases involving papilledema, fMRI of the visual pathway and particularly the retina may offer insight into the effect of papilledema on visual impairment. Retinal fMRI is still in the early stages of development and is currently performed only in animal models, still well below the resolution of optical imaging techniques. ${ }^{4-49}$ These animal experiments have already shown promise in imaging the activity of the retina in response to visual stimuli ${ }^{50}$ and can distinguish the 2 retinal layers supplied by the retinal and choroidal arteries with differing responses to hypercapnia and hyperoxia. ${ }^{51} \mathrm{fMRI}$ has been shown to detect retinal damage by evaluating decreased basal blood flow in rats. ${ }^{52}$ While not applicable to human studies, manganese-enhanced MR imaging may give additional insight into $\mathrm{ON}$ function by using the property of manganese uptake by active neurons to image neural activity. ${ }^{53-57}$

Visualization of the fiber tracts from the retina to the visual cortex is also being investigated with DTI. ${ }^{58,59}$ DTI can map optic radiations within the brain with a great deal of accuracy compared with postmortem dissections. ${ }^{60}$ Retinal ischemia in an animal study showed decreased anisotropy within the optic radiations, suggesting that DTI can detect changes in the visual pathways as early as 3 days from injury, well before the potential appearance of papilledema. ${ }^{61}$ Other experiments in rats have demonstrated decreased anisotropy within the ON following glaucoma induction and ON injury. ${ }^{54,62}$ DTI has demonstrated decreased anisotropy and increased diffusivity in children with ON hypoplasia in septooptic dysplasia. ${ }^{63}$ Disruption of these pathways leading to atrophy of distal axons projecting from the retina ${ }^{64}$ could predict visual loss from degeneration related to papilledema.

\section{CONCLUSIONS}

Papilledema represents a serious warning sign for elevated ICP and potential vision loss in a variety of clinical settings. MR imaging may facilitate its detection and demonstrate changes of elevated ICP well before the appearance of papilledema on fundoscopic examination. MR imaging signs such as flattening of the posterior sclera, dilation of the ONS, and protrusion of the optic disc head can be useful in the setting of papilledema. Although the mechanisms causing papilledema and its associated signs are not entirely clear, the role for noninvasive imaging in this clinical condition is evident. Future advances in DTI, fMRI of the retina, and high-resolution MR imaging hold the promise of demonstrating the effects of papilledema on the visual pathway in patients.

\section{REFERENCES}

1. Killer HE, Jaggi GP, Miller NR. Papilledema revisited: is its pathophysiology really understood? Clin Experiment Ophthalmol 2009;37:444-47

2. White, LH. Papilledema of ototic origin. Arch Otolaryngol $1925 ; 2: 371-78$

3. Holmes G. The prognosis in papilloedema. $\mathrm{Br} J$ Ophthalmol 1937;21:337-42

4. Hansen HC, Helmke K. Validation of the optic nerve sheath response to changing cerebrospinal fluid pressure: ultrasound findings during intrathecal infusion tests. J Neurosurg 1997;87:34-40

5. Watanabe A, Kinouchi $\mathrm{H}$, Horikoshi $\mathrm{T}$, et al. Effect of intracranial pressure on the diameter of the optic nerve sheath. J Neurosurg 2008;109:255-58

6. Brodsky MC, Vaphiades M. Magnetic resonance imaging in pseudotumor cerebri. Ophthalmology 1998;105:1686-93

7. Digre KB, Nakamoto BK, Warner JE, et al. A comparison of idiopathic intracranial hypertension with and without papilledema. Headache 2009;49:185-93

8. Gass A, Barker GJ, Riordan-Eva P, et al. MRI of the optic nerve in benign intracranial hypertension. Neuroradiology 1996;38:769-73

9. Seitz J, Held P, Strotzer M, et al. Magnetic resonance imaging in patients diagnosed with papilledema: a comparison of 6 different high-resolution T1- and T2 $\left.{ }^{*}\right)$-weighted 3-dimensional and 2-dimensional sequences. J Neuroimaging 2002;12:164-71

10. Killer HE, Laeng HR, Flammer J, et al. Architecture of arachnoid trabeculae, pillars, and septa in the subarachnoid space of the human optic nerve: anatomy and clinical considerations. Br JOphthalmol 2003;87:777-81

11. Jinkins JR, Athale S, Xiong L, et al. MR of optic papilla protrusion in patients with high intracranial pressure. AJNR Am J Neuroradiol 1996;17:665-68

12. Mashima $Y$, Oshitari K, Imamura $Y$, et al. High-resolution magnetic resonance imaging of the intraorbital optic nerve and subarachnoid space in patients with papilledema and optic atrophy. Arch Ophthalmol 1996;114:1197-203

13. Gibby WA, Cohen MS, Goldberg HI, et al. Pseudotumor cerebri: CT findings and correlation with vision loss. AJR Am J Roentgenol 1993;160:143-46

14. Atta HR, Byrne SF. The findings of standardized echography for choroidal folds. Arch Ophthalmol 1988;106:1234-41

15. Jacobson DM. Intracranial hypertension and the syndrome of acquired hyperopia with choroidal folds. J Neuroophthalmol 1995; 15:178-85

16. Lagrèze WA, Lazzaro A, Weigel M, et al. Morphometry of the retrobulbar human optic nerve: comparison between conventional sonography and ultrafast magnetic resonance sequences. Invest Ophthalmol Vis Sci 2007;48:1913-17

17. Degnan AJ, Levy LM. Pseudotumor cerebri: brief review of clinical 
syndrome and imaging findings. AJNR Am J Neuroradiol 2011;32: 1986-93. Epub 2011 Jun 16

18. Kimberly HH, Noble VE. Using MRI of the optic nerve sheath to detect elevated intracranial pressure. Crit Care 2008;12:181. Epub 2008 Sep 24

19. Geeraerts T, Newcombe VF, Coles JP, et al. Use of T2-weighted magnetic resonance imaging of the optic nerve sheath to detect raised intracranial pressure. Crit Care 2008;12:R114

20. Eliseeva NM, Serova NK, Arutiunov NV. Magnetic resonance imaging of the orbital portion of the optic nerve at different stages of papilledema [in Russian]. Vestn Oftalmol 2005;121:5-9

21. Kesler A, Yaffe D, Shapira M, et al. Optic nerve sheath enlargement and reversal of optic nerve head in pseudotumor cerebri [in Hebrew]. Harefuah 1996;130:457-59, 503

22. Trobe JD. Papilledema: the vexing issues. J Neuroophthalmol 2011;31:175-86

23. Minckler DM, Tso MO, Zimmerman LE. A light microscopic, autoradiographic study of axoplasmic transport in the optic nerve head during ocular hypotony, increased intraocular pressure, and papilledema. Am J Ophthalmol 1976;82:741-67

24. Tso MO, Hayreh SS. Optic disc edema in raised intracranial pressure. III. A pathologic study of experimental papilledema. Arch Ophthalmol 1977;95:1448-57

25. Minckler DS, Bunt AH. Axoplasmic transport in ocular hypotony and papilledema in the monkey. Arch Ophthalmol 1977;95:1430-36

26. Howden L, Giddings D, Power H, et al. Three-dimensional cerebrospinal fluid flow within the human ventricular system. Comput Methods Biomech Biomed Engin 2008;11:123-33

27. Binder DK, Horton JC, Lawton MT, et al. Idiopathic intracranial hypertension. Neurosurgery 2004;54:538-51

28. Wang SJ, Silberstein SD, Patterson S, et al. Idiopathic intracranial hypertension without papilledema: a case-control study in a headache center. Neurology 1998;51:245-49

29. Lipton HL, Michelson PE. Pseudotumor cerebri syndrome without papilledema. JAMA 1972;220:1591-92

30. Marcelis J, Silberstein SD. Idiopathic intracranial hypertension without papilledema. Arch Neurol 1991;48:392-99

31. Mathew NT, Ravishankar K, Sanin LC. Coexistence of migraine and idiopathic intracranial hypertension without papilledema. Neurology 1996;46:1226-30

32. Winner $P$, Bello L. Idiopathic intracranial hypertension in a young child without visual symptoms or signs. Headache 1996;36:574-76

33. Sher NA, Wirtschafter J, Shapiro SK, et al. Unilateral papilledema in 'benign' intracranial hypertension (pseudotumor cerebri). JAMA 1983;250:2346-47

34. To KW, Warren FA. Unilateral papilledema in pseudotumor cerebri. Arch Ophthalmol 1990;108:644-45

35. Thurtell MJ, Newman NJ, Biousse V. Visual loss without papilledema in idiopathic intracranial hypertension. J Neuroophthalmol 2010;30:96-98

36. Cordeiro MF, Nickells R, Drexler W, et al. High-resolution ocular imaging: combining advanced optics and microtechnology. Ophthalmic Surg Lasers Imaging 2009;40:480-88

37. Lagrèze WA, Gaggl $M$, Weigel $M$, et al. Retrobulbar optic nerve diameter measured by high-speed magnetic resonance imaging as a biomarker for axonal loss in glaucomatous optic atrophy. Invest Ophthalmol Vis Sci 2009;50:4223-28. Epub 2009 Apr 30

38. Hickman SJ, Brierley CM, Brex PA, et al. Continuing optic nerve atrophy following optic neuritis: a serial MRI study. Mult Scler 2002 $8: 339-42$

39. Costello FE, Goyal M. Neuroimaging in neuro-opthalmology. Neurol Clin 2010;28:757-87

40. Georgouli T, James T, Tanner S, et al. High-resolution microscopy coil MR-Eye. Eye (Lond) 2008;22:994-96. Epub 2007 Mar 2

41. Strenk SA, Semmlow JL, Strenk LM, et al. Age-related changes in human ciliary muscle and lens: a magnetic resonance imaging study. Invest Opthalmol Vis Sci 1999;40:1162-69

42. Lemke AJ, Hosten N, Wiegel T, et al. Intraocular metastases: differ- ential diagnosis from uveal melanomas with high-resolution MRI using a surface coil. Eur Radiol 2001;11:2593-601

43. Shen Q, Cheng H, Pardue MT, et al. Magnetic resonance imaging of tissue and vascular layers in the cat retina. J Magn Reson Imaging 2006;23:465-72

44. Duong TQ, Shen Q, Cheng $\mathrm{H}$, et al. Magnetic resonance imaging of anatomical layers in the cat retina. Proc Int Soc Magn Reson Med 2005;1033

45. Bridge H. Mapping the visual brain: how and why. Eye (Lond) 2011;25:291-96

46. Gore JC. Principles and practice of functional MRI of the human brain. J Clin Invest 2003;112:4-9

47. Ts'o D, Schallek J, Kwon Y, et al. Noninvasive functional imaging of the retina reveals outer retinal and hemodynamic intrinsic optical signal origins. Jpn J Ophthalmol 2009;53:334-44. Epub 2009 Sep 8

48. Duong TQ, Pardue MT, Thulé PM, et al. Layer-specific anatomical, physiological and functional MRI of the retina. NMR Biomed 2008;21:978-96

49. Duong TQ, Muir ER. Magnetic resonance imaging of the retina. Jpn J Ophthalmol 2009;53:352-67. Epub 2009 Sep 8

50. Duong TQ, Ngan SC, Ugurbil K, et al. Functional magnetic resonance imaging of the retina. Invest Ophthalmol Vis Sci 2002;43:1176-81

51. Cheng H, Nair G, Walker TA, et al. Structural and functional MRI reveals multiple retinal layers. Proc Natl Acad Sci U S A 2006;103: 17525-30. Epub 2006 Nov 6

52. Li Y, Cheng H, Shen Q, et al. Blood flow magnetic resonance imaging of retinal degeneration. Invest Ophthalmol Vis Sci 2009;50:1824-30

53. Thuen M, Singstad TE, Pedersen TB, et al. Manganese-enhanced MRI of the optic visual pathway and optic nerve injury in adult rats. J Magn Reson Imaging 2005;22:492-500

54. Thuen M, Olsen O, Berry M, et al. Combination of Mn(2+)-enhanced and diffusion tensor MR imaging gives complementary information about injury and regeneration in the adult rat optic nerve. J Magn Reson Imaging 2009;29:39-51

55. Ryu S, Brown SL, Kolozsvary A, et al. Noninvasive detection of radiation-induced optic neuropathy by manganese-enhanced MRI. Radiat Res 2002;157:500-05

56. Chan $\mathrm{KC}$, Li J, Kau P, et al. In vivo retinotopic mapping of superior colliculus using manganese-enhanced magnetic resonance imaging. Neuroimage 2011;54:389-95, Epub 2010 Jul 13

57. Berkowitz BA, Roberts R, Goebel DJ, et al. Noninvasive and simultaneous imaging of layer-specific retinal functional adaptation by manganese-enhanced MRI. Invest Ophthalmol Vis Sci 2006; 47:2668-74

58. Reinges MH, Schoth F, Coenen VA, et al. Imaging of postthalamic visual fiber tracts by anisotropic diffusion weighted MRI and diffusion tensor imaging: principles and applications. Eur J Radiol 2004;49:91-104

59. Chen J, Wang $Q$, Zhang $H$, et al. In vivo quantification of $T 1$, $T 2$, and apparent diffusion coefficient in the mouse retina at 11.74T. Magn Reson Med 2008;59:731-38

60. Sherbondy AJ, Dougherty RF, Napel S, et al. Identifying the human optic radiation using diffusion imaging and fiber tractography. $J$ Vis 2008;8:12.1-11

61. Song SK, Sun SW, Ju WK, et al. Diffusion tensor imaging detects and differentiates axon and myelin degeneration in mouse optic nerve after retinal ischemia. Neuroimage 2003;20:1714-22

62. Hui ES, Fu QL, So KF, et al. Diffusion tensor MR study of optic nerve degeneration in glaucoma. Conf Proc IEEE Eng Med Biol Soc 2007;2007:4312-15

63. Salmela MB, Cauley KA, Nickerson JP, et al. Magnetic resonance diffusion tensor imaging (MRDTI) and tractography in children with septo-optic dysplasia. Pediatr Radiol 2010;40:708-13. Epub 2009 Dec 9

64. Schoth F, Burgel U, Dorsch R, et al. Diffusion tensor imaging in acquired blind humans. Neurosci Lett 2006;398:178-82 